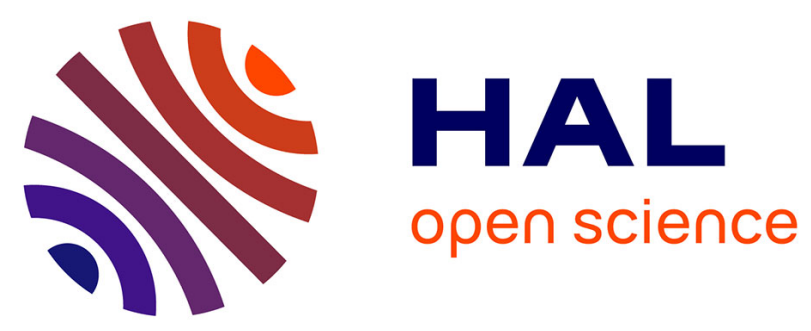

\title{
GPI 2.0: upgrading the Gemini Planet Imager
}

Jeffrey Chilcote, Quinn Konopacky, Robert de Rosa, Randall Hamper, Bruce Macintosh, Christian Marois, Marshall Perrin, Dmitry Savransky, Rémi Soummer, Jean-Pierre Véran, et al.

\section{- To cite this version:}

Jeffrey Chilcote, Quinn Konopacky, Robert de Rosa, Randall Hamper, Bruce Macintosh, et al.. GPI 2.0: upgrading the Gemini Planet Imager. Ground-based and Airborne Instrumentation for Astronomy VIII, Dec 2020, Online Only, United States. pp.333, 10.1117/12.2562578 . hal-03396792

\section{HAL Id: hal-03396792 \\ https://hal.science/hal-03396792}

Submitted on 2 Nov 2021

HAL is a multi-disciplinary open access archive for the deposit and dissemination of scientific research documents, whether they are published or not. The documents may come from teaching and research institutions in France or abroad, or from public or private research centers.
L'archive ouverte pluridisciplinaire HAL, est destinée au dépôt et à la diffusion de documents scientifiques de niveau recherche, publiés ou non, émanant des établissements d'enseignement et de recherche français ou étrangers, des laboratoires publics ou privés. 


\title{
GPI 2.0: Upgrading the Gemini Planet Imager
}

Jeffrey Chilcote ${ }^{\mathrm{a}}$, Quinn Konopacky ${ }^{\mathrm{b}}$, Robert J. De Rosa ${ }^{\mathrm{c}}$, Randall Hamper ${ }^{\mathrm{a}}$, Bruce Macintosh $^{\mathrm{d}}$, Christian Marois ${ }^{\mathrm{e}, \mathrm{f}}$, Marshall D. Perring ${ }^{\mathrm{g}}$, Dmitry Savransky ${ }^{\mathrm{h}}$, Remi Soummer ${ }^{\mathrm{g}}$, Jean-Pierre Veran ${ }^{\mathrm{e}}$, Guido Agapitoi, Arlene Aleman ${ }^{\mathrm{d}}$, S. Mark Ammons ${ }^{\mathrm{j}}$, Marco Bonaglia ${ }^{\mathrm{i}}$, Marc-Andre Boucherk ${ }^{\mathrm{k}}$, Maeve Curliss ${ }^{\mathrm{a}}$, Jennifer Dunn ${ }^{\mathrm{e}}$, Simone Esposito ${ }^{\mathrm{i}}$, Guillaume Filion ${ }^{\mathrm{k}}$, Joeleff Fitzsimmons ${ }^{\mathrm{e}}$, Isabel Kain ${ }^{\mathrm{a}, \mathrm{l}}$, Dan Kerley ${ }^{\mathrm{e}}$, Jean-Thomas Landry ${ }^{\mathrm{k}}$, Olivier Lardiere ${ }^{\mathrm{e}}$, Marie Lemoine-Busserolle ${ }^{\mathrm{m}}$, Duan $\mathrm{Li}^{\mathrm{h}}$, Mary Anne Limbach ${ }^{\mathrm{n}}$, Alex Madurowicz ${ }^{\mathrm{d}}$, Jérôme Maire $^{\mathrm{b}}$, Mamadou N'Diaye ${ }^{\mathrm{g}}$, Eric L. Nielsen ${ }^{\mathrm{d}, \mathrm{o}}$, Lisa Poyneer ${ }^{\mathrm{j}}$, Laurent Pueyo ${ }^{\mathrm{g}}$, Kaitlyn Summey ${ }^{\mathrm{h}}$, and Coleman Thomas $^{\mathrm{a}}$

${ }^{a}$ Department of Physics, University of Notre Dame, 225 Nieuwland Science Hall, Notre Dame, IN 46556, USA

${ }^{\mathrm{b}}$ Center for Astrophysics and Space Science, University of California San Diego, La Jolla, CA 92093, USA

'European Southern Observatory, Alonso de C'ordova 3107, Vitacura, Santiago, Chile

${ }^{\mathrm{d}}$ Kavli Institute for Particle Astrophysics and Cosmology, Stanford University, Stanford, CA 94305, USA

eNational Research Council of Canada Herzberg, 5071 West Saanich Rd, Victoria, BC, V9E 2E7, Canada

${ }^{\mathrm{f}}$ University of Victoria, 3800 Finnerty Road, Victoria, BC, V8P 5C2, Canada

${ }^{\mathrm{g}}$ Space Telescope Science Institute, Baltimore, MD 21218, USA

${ }^{\mathrm{h}}$ Sibley School of Mechanical and Aerospace Engineering, Cornell University, Ithaca, NY 14853, USA

${ }^{i}$ Arcetri, Largo Enrico Fermi 5, I - 50125 Florence, Italy

${ }^{j}$ Lawrence Livermore National Laboratory, Livermore, CA 94551, USA

${ }^{\mathrm{k}} 146$ Bigaouette St. Quebec City, QC, Canada, G1K 4L2

${ }^{1}$ Department of Physics, Northeastern University, Boston, MA 02115, USA

${ }^{\mathrm{m}}$ Gemini Observatory, 670 N. A'ohoku Place, Hilo, HI 96720, USA

${ }^{\mathrm{n}}$ Department of Physics and Astronomy, Texas A\&M University, 4242 TAMU, College Station, TX 77843-4242 USA

${ }^{\circ}$ Department of Astronomy, New Mexico State University, 1320 Frenger Mall, Las Cruces, NM 88003, USA

\begin{abstract}
The Gemini Planet Imager (GPI) is a dedicated high-contrast imaging facility designed for the direct detection and characterization of young Jupiter mass exoplanets. After six years of operation at Gemini South, GPI has helped establish that Jovian planets are rare at wide separations, but have higher occurrence rates at small separations. This motivates an upgrade of GPI to achieve deeper contrasts, especially at small inner working angles, while leveraging its current capabilities. GPI has been funded to undergo a major science-driven upgrade as part of a relocation to Gemini North (GN). Gemini plans to remove GPI at the end of 2020A. We present the status of the proposed upgrades to GPI including a EMCCD-based pyramid wavefront sensor, broadband low spectral resolution prisms and new apodized-pupil Lyot coronagraph designs. We discuss the expected
\end{abstract}

Further author information: (Send correspondence to Jeffrey K. Chilcote)

Jeffrey K. Chilcote: E-mail: jchilcote@nd.edu 
performance improvements in the context of GPI 2.0's enhanced science capabilities which are scheduled to be made available at GN in 2022 .

Keywords: Adaptive optics; extrasolar planets; coronagraphy; integral field spectrograph

\section{INTRODUCTION}

Since the discovery of 51 Pegasi in $1995,{ }^{1}$ the search for and discovery of extrasolar planets has dramatically changed our understanding of planetary formation and the place where our solar system stands with respect to other solar systems in the galaxy. While thousands of exoplanets have now been discovered only a few of the most massive or widest separation ones have been observed spectroscopically. Direct imaging is expected to play an increasingly significant role in the future. ${ }^{2}$ Direct imaging allows for the discovery of planets on solar system-scale orbits, provides new insight into the formation and characteristics of extrasolar systems, and enables direct spectroscopic observations of their atmospheres.

The Gemini Planet Imager (GPI) is a facility class instrument designed to address the fundamental goal of directly detecting and observing exoplanets (Figure 1). GPI was designed and built to directly image and spectroscopically characterize young, Jupiter-sized, self-luminous extrasolar planets and search for circumstellar debris disks that are sculpted by planetary systems. The primary motivation for creating GPI was to measure the frequency and distribution of wide-orbit, giant planets. While designed for either Gemini North or Gemini South, GPI was installed at Gemini South in the fall of $2013 .^{3}$

\subsection{Overview of exoplanet science with GPI}

The original GPI consisted of an adaptive optics (AO) system, apodized-pupil Lyot coronograph (APLC), a precision infrared wavefront sensor (CAL), and a near-IR integral field spectrograph (IFS). The AO system consists of a 4096-acuator microelectro-mechanical (MEMS) deformable mirror, a CILAS 11 actuator diameter piezoelectric DM in a woofer-tweeter configuration, and a Shack-Hartmann WFS design with a Lincoln Labs CCID-66 sensor. ${ }^{3,4}$ GPI uses an APLC to suppress coherent light from the central star. ${ }^{5}$ GPI's science instrument is an IFS with 192x192 spatial pixels dispersed through a prism to provide a resolving power of $\mathrm{R}=\sim 30-100$ depending upon the band. The GPI IFS has 5 individual filters in $\mathrm{Y}, \mathrm{J}, \mathrm{H}$, and 2 in K-band (split into overlapping segments). The IFS further incorporates a Wollaston prism to allow for polarization measurements but only in broad band. ${ }^{6,7}$

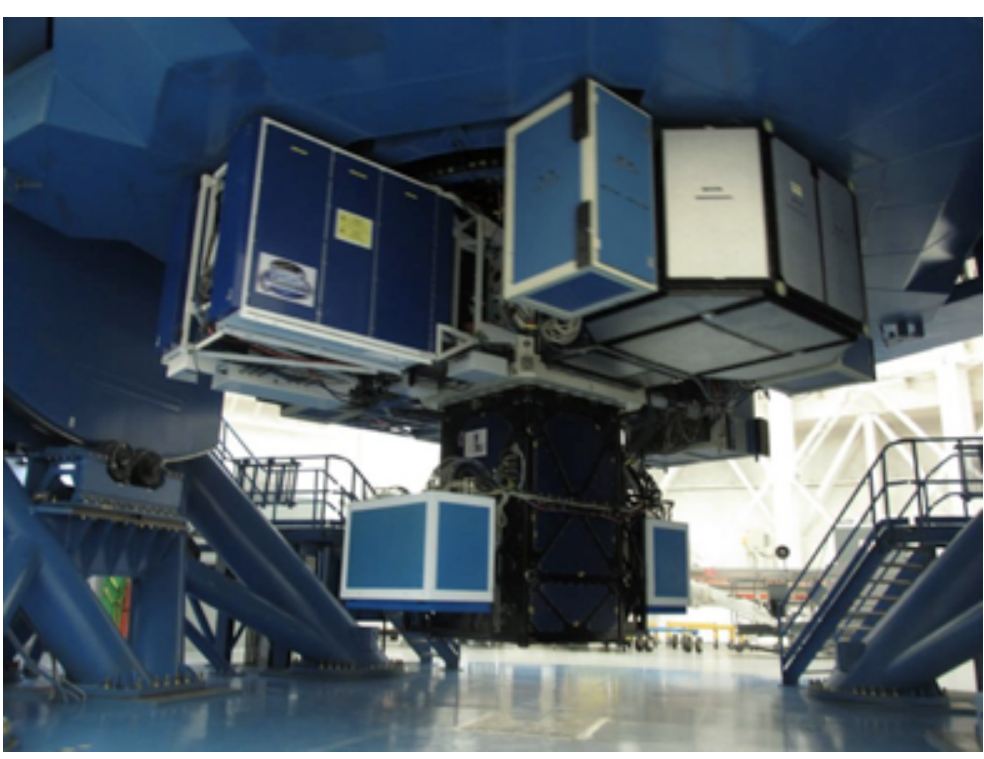

Figure 1: GPI (bottom) mounted on the Cassegrain mount of the Gemini South telescope, Chile.

In this work, we will present the upgrades being made to GPI known as GPI 2.0. In Section 2 we discuss the current capabilities of GPI, its historical usage, and significant results. In Section 3 we discuss the next generation science drivers which drove the hardware upgrades of GPI. And finally in Section 4 we present the current funded upgrades being planed for GPI known as GPI 2.0. 


\section{SUMMARY OF GPI 1.0}

GPI was installed at Gemini South in 2013 and has been in routine operations since the 2014B semester. In addition to the large-scale exoplanet survey (GPIES), there have been two Large and Long Programs (LLP) which span multiple semesters, 90 accepted regular proposals, 8 Director's Discretionary Time, and 14 though Gemini's Fast Turnaround program. These proposals were from 58 unique principal investigators. Based on their titles, about half of the accepted programs are related to exoplanet detection and/or characterization, a third are exploiting GPI's polarimetric capability to characterize circumstellar material around both young and more evolved stars. The remaining programs are focused on topics ranging from astrometric monitoring of young binaries to characterizing degenerate companions. Excluding time allocated for the GPIES campaign and science verification programs, 848 hours have been queued to GPI observations between 2014B and 2020A. The GPIES campaign was proposed as an 890 hour survey which operated exclusively in priority visitor mode.

GPI has been highly scientifically productive, with 73 peer-reviewed articles by many users across the US and international partners. The papers produced by GPI have been cited 2661 times (an instrument h-index of 30). GPI publications have included several high-impact results such as the discovery of $51 \mathrm{Eri} \mathrm{b},{ }^{8}$ the closest analog to Jupiter in terms of mass and semi-major axis amongst all directly imaged planets. GPI observations have been used to constrain the orbital parameters of the planet ${ }^{9}$ and the atmospheric composition and fundamental properties of the planet. ${ }^{10}$ In addition to the discovery of a new brown dwarf companion to HR $2562,{ }^{11}$ GPI has been used to spectroscopically characterize previously known substellar companions, ${ }^{12-18}$ providing new insights into the diversity of substellar companion atmospheres at a range of masses and ages. The architectures of planetary systems have also been investigated, with GPI observations providing indirect evidence of the presence of additional companions within the HD 95086 system. ${ }^{19}$

In an initial analysis of the exoplanet population resolved, GPI measured the frequency of wide-orbit (10$100 \mathrm{au})$ massive $\left(5-13 \mathrm{M}_{\mathrm{Jup}}\right)$ planets to be $11.4 \pm 5.5 \%{ }^{20}$ The occurrence rate is strongly dependent on stellar mass, with all detected planet-hosting stars being above $1.5 \mathrm{M}_{\odot}$. These results show that we cannot simply extrapolate outwards the planet population detected via radial velocity; either there is a break in the planet distribution between 5-10 au, or most giant planets at wide separations formed through a low-entropy formation process resulting in a low initial luminosity. Combined with Doppler and microlensing rates, this indicates that systems with giant planets are rare - perhaps 0.25 such planets per star. GPI's spectral and polarimetric capabilities have also been used to search for and characterize transition disks ${ }^{21-25}$ and debris disks ${ }^{26-28}$ around nearby young stars. These observations have been use to determine the geometry and composition of circumstellar disks $^{29}$ and postulate the presence of unseen planetary-mass companions based on asymmetries or structure resolved within the disk..$^{30,31}$

\section{NEXT GENERATION SCIENCE GOALS}

GPI has been in nearly continuous operation since 2013 without a significant maintenance overhaul to its internal components. Additionally, in 2018, GPI was being reviewed for a possible relocation from Gemini South to Gemini North. ${ }^{32,33}$ Any move from Chile to Hawaii would require significant disassembly of the instrument and an inspection of its internal components to ensure the safety and reliability of the instrument as part of a move. It was recognized that this relocation and maintenance cycle would be an ideal time to improve the instrument with hardware upgrades to address the next generation of science requirements. To consider which components would be most valuable to upgrade, a series of science cases were considered to drive the hardware upgrades in GPI.

\subsection{Imaging Exoplanets around Nearby Young Stars}

The first science area identified was to significantly improve GPI's sensitivity to planetary-mass companions in the 4-10 au range around nearby stars, straddling the peak of the distribution of giant planets measured for solar-type stars, and enabling a measurement of the location of this peak for more massive stars. Currently, the only unambiguous detection of an exoplanet consistent with the predictions of a "cold-start" formation scenario is 51 Eridani b. ${ }^{8,10}$ The planet lies right at the sensitivity limit of GPIES, with observations of 51 Eri b analogues only possible around $\sim 16$ other stars in the survey. The next evolution of GPI must provide an improvement on 

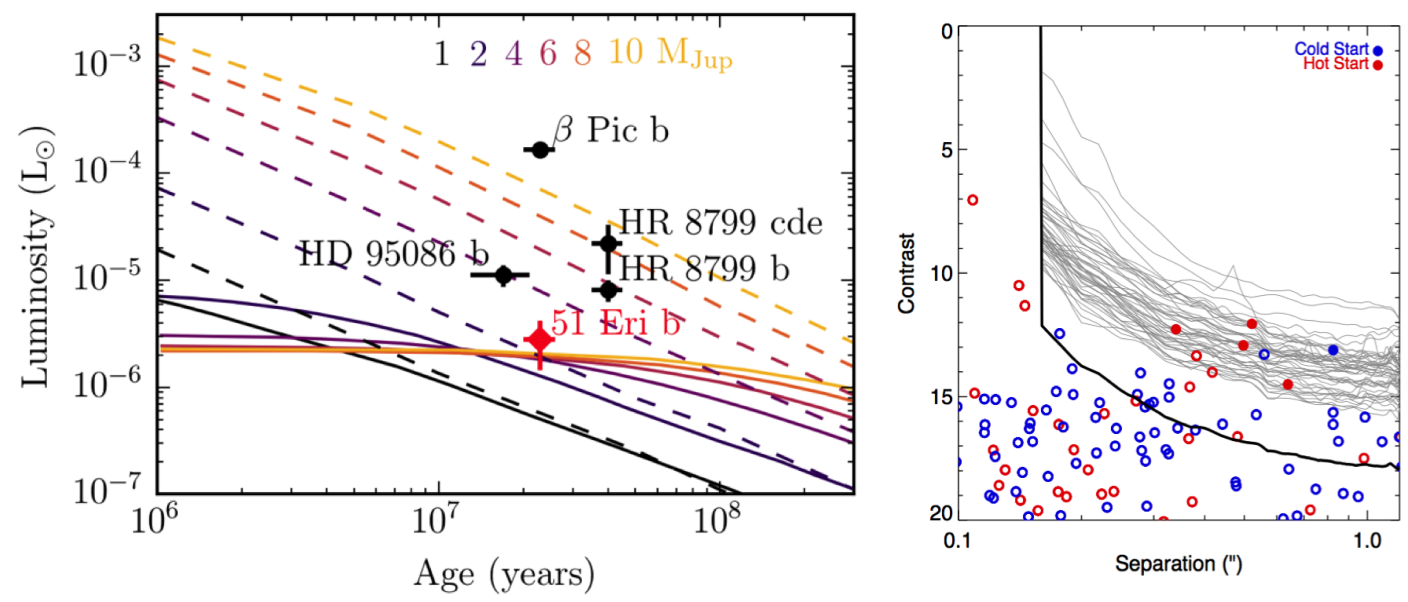

Figure 2: Left: Hot-start (dashed) vs cold-start evolutionary models for 1-10Mj with age vs luminosity. ${ }^{34}$ Several known planets indicated. Right: In gray are typical GPI contrast curves with the present instrument. In black is a prediction of improving GPI's contrast by 3.2 magnitudes. The planet population is modeled using a distribution of planets consistent with radial velocity and direct imaging surveys. Filled circles represent planets that could be detected with the current observations, open circles would fall below the contrast curve for their particular host star.

the constraints of the distribution of giant planets as a function of semi-major axis, and to differentiate between the two canonical planet formation processes; the low-entropy "cold-start" models ${ }^{34}$ and the high-entropy "hotstart" models. ${ }^{35}$ "Cold-start" evolutionary models predict a similar luminosity for exoplanets of 2-13 M Jup and between 10-100 Myrs. This degeneracy exists at the youngest ages, after which the lowest-mass planets cool more rapidly to match the predictions of the "hot-start" formation models. Models suggest that a modest increase in contrast of 1-2 magnitudes would lead to a significant increase in the area of the mass/semi-major axis phase space that GPI could probe. As seen in Figure 2, while cold start models predict low-luminosity planets, the overlap of models at $\sim 2 \times 10^{-6} \mathrm{~L}_{\odot}$ means a small increase in achieved contrast around a young star will rapidly switch from no sensitivity to cold start planets to being sensitive to cold start planets between 2 and $10 \mathrm{M}_{\text {Jup }}$. This is a stark difference from hot start planets, where luminosity increases gradually and monotonically with mass.

In addition to improving sensitivity to the lowest-mass giant planets around the youngest stars, increasing the contrast will allow for searches for higher-mass planets around stars that are somewhat older (300-500 Myr) but that are significantly closer to the Sun $(20-30 \mathrm{pc})$. The proximity of these stars will allow an upgraded GPI to probe separations that are consistent with the location of the peak of the occurrence rate distribution as measured for Solar-type stars from previous radial velocity (RV) surveys ${ }^{36}$ allowing for better constraints on the distribution of the separation distribution of giant planets and providing further insight into the evolution and atmospheric properties of these objects as they radiate away their formative heat.

\subsection{Probing planet formation in disks}

The opportunity of changing hemispheres will enable new probes of the youngest stars in two regions- Upper Scorpius (Upper Sco) and Taurus, which both host numerous young, bright, nearby stars. With the improved performance of an upgraded $\mathrm{AO}$ system on faint stars plus new coronagraphs that enable probes of the tightest inner working angles, surveys of these two regions could reveal a number of newly formed planets that can shed light on distribution and atmospheric properties of targets shortly after formation. By identifying these sources and comparing their demographics with those of the adolescent planets probed by current direct imaging surveys, we can constrain important processes that occur in the early lifetimes of planets, such as migration timescales or atmospheric chemistry evolution. Due to the distance to these targets, GPI will not be sensitive to the closest regions to the star. However, given the youth of these sources, GPI will be broadly sensitive to a range of hot 


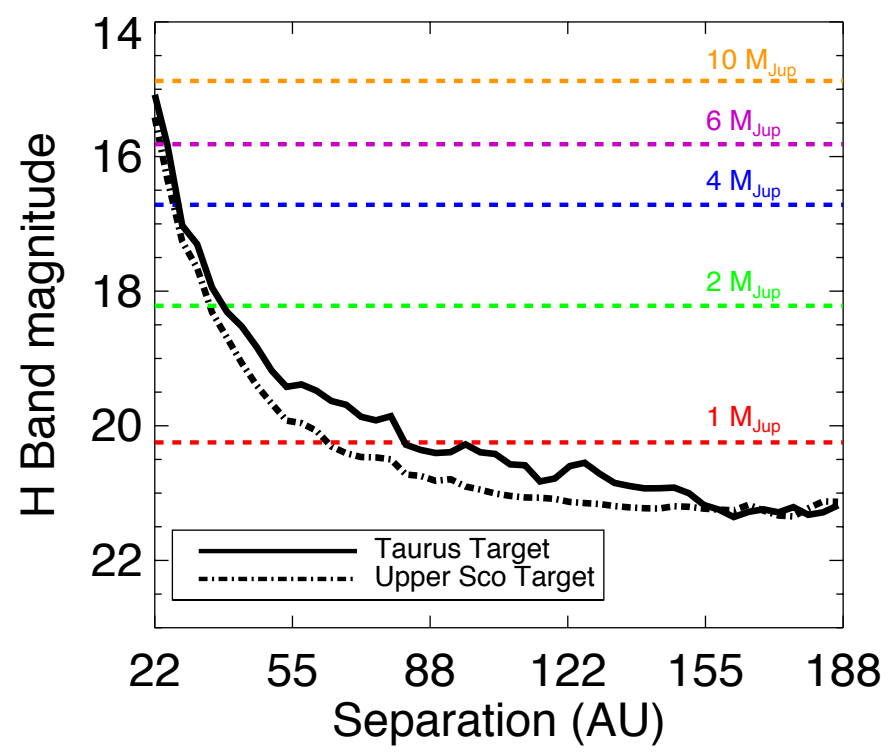

Figure 3: GPI 2.0 sensitivity predictions for very young planets in Upper Sco and Taurus. Contrast curves for two example targets with similar H-band magnitudes but different I band magnitudes are shown. These curves are based on predictions from current GPI performance. The predicted brightnesses of hot-start planets at these ages are shown as dashed colored lines. Note that we will be sensitive to planets as small as $4 \mathrm{M}_{\mathrm{Jup}}$ at all separations and down to $1 \mathrm{M}_{\mathrm{Jup}}$ at wider separations.

start planet masses. Figure 3 shows GPI's expected performance on two example targets at 145 pc in Upper Sco and Taurus, each of which have $H=7.5$ with $I=7.5$ and $I=10.5$, respectively.

While surveying these sources for planetary companions, their disks can be probed in detail. There is a wide variety of planet formation science that can be explored by high contrast imaging of transition disks. Previous studies of transition disks have explored their morphologies, with a wide range of structures such as gaps or spirals thought to be caused by ongoing interactions with forming planets. To enable this science, GPI will require being able to operate on fainter stars then it has in the past. With an improved magnitude limit for GPI 2.0, a number of transition disk hosts become observable, allowing for a detailed study of a range of sources as a function of age and host star mass. This is particularly important for the transition disks in Taurus - the very youngest available to us, fainter and only accessible from the Northern hemisphere.

\subsection{Asteroids \& Solar System Objects}

Asteroids in our solar system are metallic, rocky and icy objects ranging in size from a few meters to a few hundred kilometers. While we have constraints for the surface composition, albedo and rotation rates for many main-belt asteroids the 3-D shape, crater distribution and density have only been measured for a limited number of bodies. Characterizing these physical properties would allow us to address entirely new questions regarding the earliest stages of planetesimal formation and their subsequent collisional and dynamical evolution. Operating GPI at a lower visible magnitude limit for its AO system while maintaining high contrast capabilities will allow expansion of the number of targets studied. For example, if the AO system were able to achieve a limiting magnitude of $\mathrm{V}=14$ then $\sim 1300$ objects would be available for study. GPI brings with it the ability to provide spectroscopy allowing for an observation of the mafic bands to characterize the surface composition. Further, GPI's AO capabilities are needed in order to study the properties of companion asteroids, not only single asteroids.

\subsection{Planet Variability}

Understanding the atmospheres of exoplanets is one of the key goals of direct imaging. Current models of the characterization of exoplanets such as HR8799 c,d,e and $\beta$ Pic b indicate the presence of both non-equilibrium 

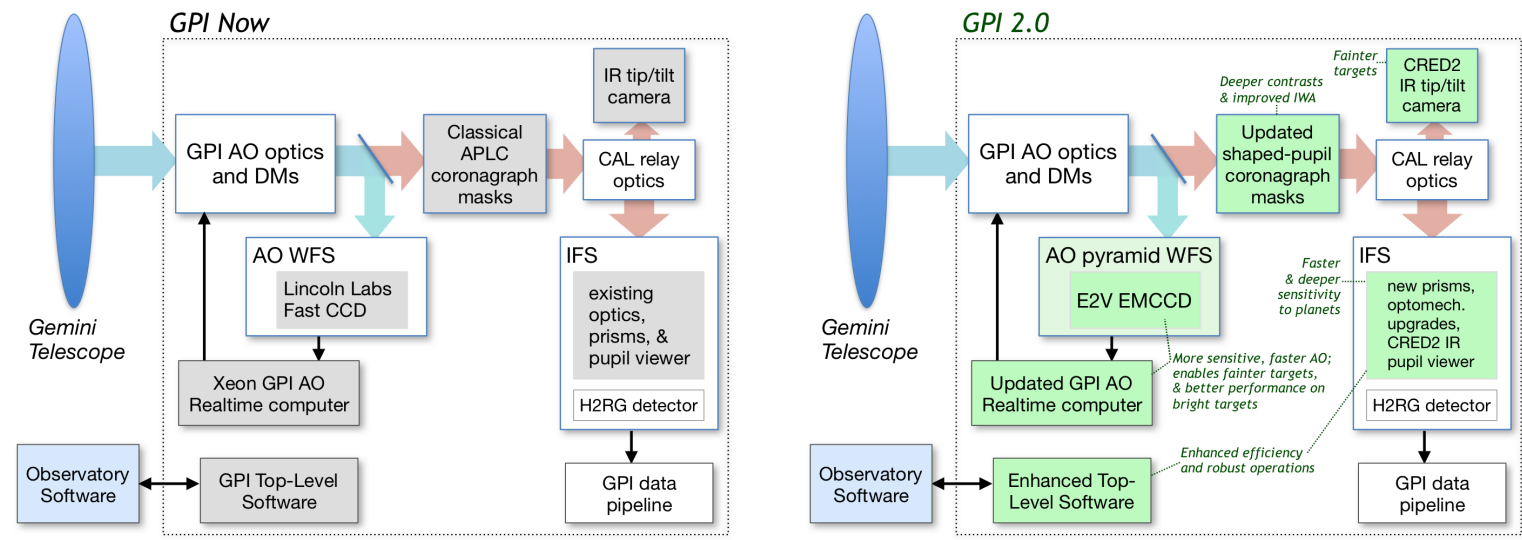

Figure 4: Schematic of GPI subsystems with the original as built system (Left) and the upgraded subsystems (Right)

chemistry and patchy clouds, which can confound inferences of their composition. ${ }^{37}$ Patchy clouds are often indicated as important factors in understanding the history and composition of these planetary atmospheres. As planets rotate, variable cloud cover induces modulation in the flux of a planet. This variability has been used to measure the rotation rates and study the atmospheres of planetary-mass bodies and brown dwarfs. In order to study this variability for a significant number of targets, it is estimated that GPI would need to improve its capabilities to achieve $1 \%$ photometric stability on the brightest targets.

\section{GPI 2.0 INSTRUMENT UPGRADES}

\subsection{AO Upgrade: Improving contrast and limiting magnitude}

The original adaptive optics (AO) subsystem in GPI used a spatially-filtered, Shack-Hartmann wavefront sensor (WFS), designed around a Lincoln Labs CCID-66 sensor, along with a CILAS 95-actuator piezoelectric deformable mirror (DM) and a Boston Micromachines 4096-actuator micro-electro-mechanical (MEMS) DM. The original GPI science case focused on observations of moderate-mass stars in nearby ( $\lesssim 60 \mathrm{pc})$ moving groups. Because the vast majority of these targets are brighter than $I=9 \mathrm{mag}$, the AO wavefront sensor (AOWFS) was specified with a limiting magnitude of $I=9$, with a goal of $I=10$. A conventional CCD detector was used rather than the novel (at the time) near-zero-noise EMCCD.

GPI 2.0 will be removing the existing spatially-filtered, Shack-Hartmann wavefront sensor and replacing it with a pyramid WFS leveraging the existing design studies performed for NFIRAOS. ${ }^{38}$ NRC Herzberg Astronomy and Astrophysics is leading the design of the Pyramid WFS and construction, alignment and testing is being lead by the University of California, San Diego. This upgrade is being designed as an independent sub-bench to the existing $\mathrm{AO}$ bench and will replace the optical leg after the original system's beam splitter. The upgraded design features four total mirrors, two of which are fold mirrors and two of which are field steering mirrors, one of which will also provide the pyramid's modulation. The upgraded $\mathrm{AO}$ system will operate of $2 \mathrm{kHz}$ and will incorporate NUVU's HNü 240 which uses an E2V CCD 220. To estimate the Phyramid WFS's performance in GPI, the team has used PASSATA for AO simulations ${ }^{39}$ and simulations of performance described in Madurowicz et al. ${ }^{40}$ Simulations of End-to-End AO performance is estimated to produce a Strehl ratio in H-band of at least 0.9 until 12th magnitude and to continue to operate, albeit with decreased performance, past 14th magnitude. While the Gemini North M2 print-through ${ }^{41}$ and the GPI MEMS DM dead and coupled actuators ${ }^{42}$ will affect final GPI contrast and science performance, they do not inhibit the simulated operations of the new GPI Pyramid WFS provided appropriately applied mitigation strategies.

\subsection{Coronagraph Upgrade: Improving inner working angle}



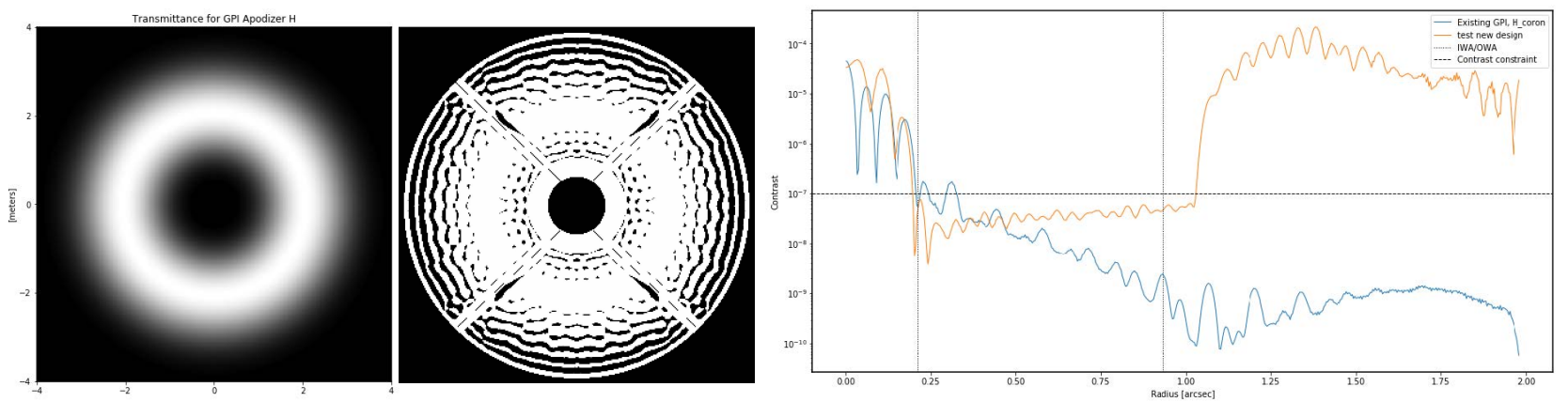

Figure 6: Left: GPI's current H-band prolate apodization function. The throughput of this design is only $46 \%$. Center: This pupil plane function is generated using current techniques, but using the same constraints defined as part of the original GPI design. The throughput of this design is $76 \%$. Right: The radial profile contrast constraint assuming no residual wavefront error from AO system.

To obtain deeper contrasts (especially close to the star), we will replace GPI's apodized pupil Lyot coronagraph masks ${ }^{43,44}$ with a new generation of designs using the latest techniques. This upgrade takes advantage of a decade of technology and concept development since the original GPI coronagraphs, driven by missions such as NASA's Nancy Grace Roman Space Telescope Coronagraphic Imager (CGI). GPI currently uses a set of APLC masks designed to reach a raw contrast of $10^{-7}$ at 0.2 arcsec in $20 \%$ broadband light. A continuous prolate apodization was selected to optimize contrast and achieve quasi-achromatic solutions over the needed $20 \%$ bandpass. ${ }^{45}$ However, because there was only a single degree of freedom in the definition of that apodizer, the raw performance was limited both in inner working angle (IWA) and contrast. Since that GPI coronagraph design circa 2008, the theory of APLC coronagraph design has considerably advanced, in particular to work with binary,

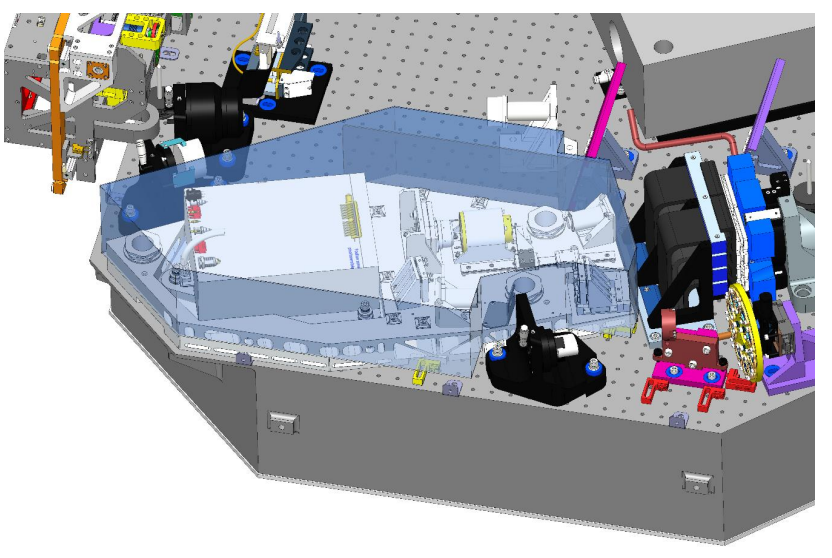

Figure 5: The layout of the upgraded Pyramid based $\mathrm{AO}$ bench. $^{38}$ Inside of the blue box is the location of the new AO sub-plate, new AO optical components, and PYWFS. Outside of the blue box is the existing GPI optical components which will remain.

shaped-pupil apodizations generated by full numerical optimization. ${ }^{46-48}$ There are now solutions to produce extremely strong diffraction suppression, in broadband (18\%), with relatively small inner working angle $(3 \lambda / D)$, and for complex telescopes including segmented pupils. ${ }^{47}$ Original studies applying these techniques to improve GPI were were published in N'Diaye et al. ${ }^{46}$ GPI 2.0 will retain the same focal plane masks as the original GPI but pair these with new pupil plane masks. GPI 1.0 had 10 pupil plane mask options and 8 focal plane masks. In GPI 2.0, 3 of the pupil plane masks will be retained for engineering purposes and legacy in the most widely used H-band mode. The NRM mask will be relocated to the IFS. The legacy H-band mode will enable a comparison to historical performance of GPI. The remaining 7 slots will be used in conjunction with the existing focal plane masks, at their existing physical sizes, to offer a new generation of APLC coronograph designs for GPI. This will allow new options such as better inner working angles or higher throughput to GPI's IFS. For example, using the existing design parameters for $\mathrm{GPI}^{5}$ new, optimized designs were created that have a $76 \%$ transmition vs. the existing designs which only have a $46 \%$ transmission (Figure 6). These improvements will become important as GPI looks towards observing fainter stars now enabled by the upgraded AO system. The variety of slots available to GPI will enable optimizations of IWA, thoughput, alignment tolerance, and contrast for multiple science cases. 


\subsection{CAL 2.0}

The NRC Herzberg Astronomy and Astrophysics research centre will replace the high-order WFS sub-system of GPI. Originally utilizing a dual-arm interferometer design, this system was implemented to measure and to correct for remaining uncorrected aberrations as seen on the science camera at a speed of a few $\mathrm{Hz}$, but has never worked as intended due to vibrations. CAL 2.0's main focus is the Self Coherent Camera (SCC). The SCC uses a common-path interferometer design to enable focal plane wavefront sensing and control simply by acquiring science camera images showing fringes, which simultaneously allows (i) the measurement of the science focal plane electric field (a standard focal plane imaging science camera only measures intensity), and (ii) the removal of uncorrected stellar noise by post-processing and/or by a feedback loop to the adaptive optics (AO) deformable mirror. The design and overview of CAL 2.0 is discussed in depth in Marois et al. ${ }^{49}$

\subsection{IFS Upgrade: single-shot JHK for efficient surveys and monitoring}

The GPI IFS ${ }^{6,7}$ is a cryogenic instrument sensitive from $0.95-2.4 \mu \mathrm{m}$ that measures an image at multiple wavelengths simultaneously. The GPI 2.0 upgrade will modify the prisms inside of the IFS to enable new observing resolutions. We will add a new set of prisms to the IFS to enable a low-spectral-resolution observing mode $(R \sim 10)$ and a higher resolution mode in line with GPI's original resolution but even across the filter bands. The design of these prisms, observing modes, and resolutions enabled by GPI are discussed in depth in Limbach et al. ${ }^{50}$ This mode is inspired by the one currently implemented on the Japanese instrument CHARIS, ${ }^{51}$ and will enable observations at a lower spectral resolution but broader wavelength range then GPI 1.0. This mode will operate from Y-K band simultaneously. By decreasing the resolution of GPI, around faint stars there will be increased sensitivity for discovery of faint companions. Reduced spectral resolution across a broader range will concentrate more light into fewer pixels allowing for a higher signal-to-noise ratio. This is ideal when searching for new and fainter planets to maximize observing efficiency. Secondly, the broader spectral range will provide increased leverage against several kinds of calibration systematics and enable GPI to improve its photometric calibration between spectra. GPI was designed to have low chromaticity, and sources the existing chromaticity in GPI are being investigated. The Spectral Differential Imaging (SDI) technique relies on the wavelength dependence of speckles; expanding the spectral range with GPI will increase its effectiveness. Increased sensitivity from the low-res mode is closely complementary to increased contrast in enabling detection of fainter planets undetectable with current instrumentation. In parallel with adding the low-res mode, the high resolution prisms will also be upgraded to enable all of $K$-band to be observed at the same time, reducing the amount of telescope time needed to observe all of $K$-band by more than $50 \%$. In the original GPI design, modeling showed $\mathrm{R}>40$ was needed for good temperature estimation from a single band. Given the prism materials available at the time, in order to obtain an R 40 in H-band, a higher resolution resulted in K-band and a lower in Y-band. Analysis using current model grids has shown that a lower resolution of around $\mathrm{R} \sim 10$ is sufficient combined with the wider spectral range to provide accurate temperature estimates and is useful in separating background objects.

\subsection{GPI Operational \& Usability Upgrade}

While GPI's reliability has been very good, and the instrument is well integrated with observatory systems, five years of operations at Gemini South has shown there are potential upgrades which would make the entire system more robust and user friendly. Currently, the GPI operator checklist contains approximately ten discrete user actions that could be removed via software remediation. Additional error checking in the alignment and calibration process will reduce the number of steps and remove multiple human interventions required in the case of an alignment failure. Additionally, GPI 2.0 will involve an update of the open loop models which are used for internal alignment based upon temperature and telescope angle. Additionally, GPI's high-level control library was implemented originally in Interactive Data Language (IDL). GPI 2.0 will port this library to the more modern language of Python to improve reliability of future instrument operations.

\section{CONCLUSION}

Many of the design decisions which went into the construction of GPI were made in 2004. The technology and field of ExAO systems has greatly advanced in the intervening years. Since GPI started standard science operations on sky in 2014, it has not had any major hardware changes to the instrument. The lack of hardware 
changes had lead to the stability and reliability that has helped GPI to achieve its major science goals and to provide a platform to cutting edge science. Using the results of the previous decade, and in-order to stay competitive with cutting edge instruments today, GPI is undertaking a significant upgrade to pursue a science driven upgrade of its hardware. This will accompany a move from Gemini South to Gemini North. These these hardware changes and upgrade GPI to enable cutting edge science from Gemini North for many years to come.

\section{ACKNOWLEDGMENTS}

This material is based upon work supported by the National Science Foundation under Grant No. AST1920180, AST-1411868, and AST-1518332. Significant portions of this work are supported by the Heising-Simons Foundation. The authors were supported in part by NASA NNX14AJ80G, NNX15AC89G, NNX15AD95G, NSSC17K0535, and NNG16PJ24C. This work has been supported by the STScI Director's Discretionary Research Fund. Research benefited from the Gemini Observatory, operated by AURA for NSF and the Gemini consortium. This work benefited from NASA's Nexus for Exoplanet System Science (NExSS) research coordination network sponsored by NASA's Science Mission Directorate.

\section{REFERENCES}

[1] Mayor, M. and Queloz, D., "A Jupiter-mass companion to a solar-type star," Nature 378, 355-359 (Nov. 1995).

[2] National Academies of Sciences, Engineering, and Medicine, [Exoplanet Science Strategy], The National Academies Press, Washington, DC (2018).

[3] Macintosh, B., Graham, J. R., Ingraham, P., Konopacky, Q., Marois, C., Perrin, M., Poyneer, L., Bauman, B., Barman, T., Burrows, A. S., Cardwell, A., Chilcote, J., De Rosa, R. J., Dillon, D., Doyon, R., Dunn, J., Erikson, D., Fitzgerald, M. P., Gavel, D., Goodsell, S., Hartung, M., Hibon, P., Kalas, P., Larkin, J., Maire, J., Marchis, F., Marley, M. S., McBride, J., Millar-Blanchaer, M., Morzinski, K., Norton, A., Oppenheimer, B. R., Palmer, D., Patience, J., Pueyo, L., Rantakyro, F., Sadakuni, N., Saddlemyer, L., Savransky, D., Serio, A., Soummer, R., Sivaramakrishnan, A., Song, I., Thomas, S., Wallace, J. K., Wiktorowicz, S., and Wolff, S., "First light of the Gemini Planet Imager," Proceedings of the National Academy of Science 111, 12661-12666 (Sept. 2014).

[4] Poyneer, L. A., De Rosa, R. J., Macintosh, B., Palmer, D. W., Perrin, M. D., Sadakuni, N., Savransky, D., Bauman, B., Cardwell, A., Chilcote, J. K., Dillon, D., Gavel, D., Goodsell, S. J., Hartung, M., Hibon, P., Rantakyrö, F. T., Thomas, S., and Veran, J.-P., "On-sky performance during verification and commissioning of the Gemini Planet Imager's adaptive optics system," in [Adaptive Optics Systems IV], Proc. SPIE 9148, 91480K (July 2014).

[5] Soummer, R., Sivaramakrishnan, A., Pueyo, L., Macintosh, B., and Oppenheimer, B. R., "Apodized Pupil Lyot Coronagraphs for Arbitrary Apertures. III. Quasi-achromatic Solutions," Astrophysical Journal 729, 144 (Mar. 2011).

[6] Larkin, J. E., Chilcote, J. K., Aliado, T., Bauman, B. J., Brims, G., Canfield, J. M., Cardwell, A., Dillon, D., Doyon, R., Dunn, J., Fitzgerald, M. P., Graham, J. R., Goodsell, S., Hartung, M., Hibon, P., Ingraham, P., Johnson, C. A., Kress, E., Konopacky, Q. M., Macintosh, B. A., Magnone, K. G., Maire, J., McLean, I. S., Palmer, D., Perrin, M. D., Quiroz, C., Rantakyrö, F., Sadakuni, N., Saddlemyer, L., Serio, A., Thibault, S., Thomas, S. J., Vallee, P., and Weiss, J. L., "The integral field spectrograph for the Gemini planet imager," in [Ground-based and Airborne Instrumentation for Astronomy V], Proc. SPIE 9147, 91471K (July 2014).

[7] Chilcote, J. K., Larkin, J. E., Maire, J., Perrin, M. D., Fitzgerald, M. P., Doyon, R., Thibault, S., Bauman, B., Macintosh, B. A., Graham, J. R., and Saddlemyer, L., "Performance of the integral field spectrograph for the Gemini Planet Imager," in [Society of Photo-Optical Instrumentation Engineers (SPIE) Conference Series], Society of Photo-Optical Instrumentation Engineers (SPIE) Conference Series 8446 (Sept. 2012).

[8] Macintosh, B., Graham, J. R., Barman, T., De Rosa, R. J., Konopacky, Q., Marley, M. S., Marois, C., Nielsen, E. L., Pueyo, L., Rajan, A., Rameau, J., Saumon, D., Wang, J. J., Ammons, M., Arriaga, P., Artigau, E., Beckwith, S., Brewster, J., Bruzzone, S., Bulger, J., Burningham, B., Burrows, A. S., Chen, C., Duchene, G., Esposito, T. M., Fabrycky, D., Fitzgerald, M. P., Follette, K. B., Fortney, J. J., Gerard, B., 
Goodsell, S., Greenbaum, A. Z., Hibon, P., Hinkley, S., Hufford, T., Hung, L.-W., Ingraham, P., JohnsonGroh, M., Kalas, P., Lafreniere, D., Larkin, J. E., Lee, J., Line, M., Long, D., Maire, J., Marchis, F., Matthews, B. C., Max, C. E., Metchev, S., Millar-Blanchaer, M. A., Mittal, T., Morley, C. V., Morzinski, K. M., Murray-Clay, R., Oppenheimer, R., Palmer, D. W., Patel, R., Patience, J., Perrin, M. D., Poyneer, L. A., Rafikov, R. R., Rantakyro, F. T., Rice, E., Rojo, P., Rudy, A. R., Ruffio, J.-B., Ruiz, M. T., Sadakuni, N., Saddlemyer, L., Salama, M., Savransky, D., Schneider, A. C., Sivaramakrishnan, A., Song, I., Soummer, R., Thomas, S., Vasisht, G., Wallace, J. K., Ward-Duong, K., Wiktorowicz, S. J., Wolff, S. G., and Zuckerman, B., "Discovery and spectroscopy of the young jovian planet 51 Eri b with the Gemini Planet Imager.," Science 350, 64-67 (2015).

[9] De Rosa, R. J., Nielsen, E. L., Blunt, S. C., Graham, J. R., Konopacky, Q. M., Marois, C., Pueyo, L., Rameau, J., Ryan, D. M., Wang, J. J., Bailey, V., Chontos, A., Fabrycky, D. C., Follette, K. B., Macintosh, B., Marchis, F., Ammons, S. M., Arriaga, P., Chilcote, J. K., Cotten, T. H., Doyon, R., Duchêne, G., Esposito, T. M., Fitzgerald, M. P., Gerard, B., Goodsell, S. J., Greenbaum, A. Z., Hibon, P., Ingraham, P., Johnson-Groh, M., Kalas, P. G., Lafrenière, D., Maire, J., Metchev, S., Millar-Blanchaer, M. A., Morzinski, K. M., Oppenheimer, R., Patel, R. I., Patience, J. L., Perrin, M. D., Rajan, A., Rantakyrö, F. T., Ruffio, J.-B., Schneider, A. C., Sivaramakrishnan, A., Song, I., Tran, D., Vasisht, G., Ward-Duong, K., and Wolff, S. G., "Astrometric Confirmation and Preliminary Orbital Parameters of the Young Exoplanet 51 Eridani b with the Gemini Planet Imager," Astrophysical Journal Letters 814, L3 (Nov. 2015).

[10] Rajan, A., Rameau, J., De Rosa, R. J., Marley, M. S., Graham, J. R., Macintosh, B., Marois, C., Morley, C., Patience, J., Pueyo, L., Saumon, D., Ward-Duong, K., Ammons, S. M., Arriaga, P., Bailey, V. P., Barman, T., Bulger, J., Burrows, A. S., Chilcote, J., Cotten, T., Czekala, I., Doyon, R., Duchêne, G., Esposito, T. M., Fitzgerald, M. P., Follette, K. B., Fortney, J. J., Goodsell, S. J., Greenbaum, A. Z., Hibon, P., Hung, L.-W., Ingraham, P., Johnson-Groh, M., Kalas, P., Konopacky, Q., Lafrenière, D., Larkin, J. E., Maire, J., Marchis, F., Metchev, S., Millar-Blanchaer, M. A., Morzinski, K. M., Nielsen, E. L., Oppenheimer, R., Palmer, D., Patel, R. I., Perrin, M., Poyneer, L., Rantakyrö, F. T., Ruffio, J.-B., Savransky, D., Schneider, A. C., Sivaramakrishnan, A., Song, I., Soummer, R., Thomas, S., Vasisht, G., Wallace, J. K., Wang, J. J., Wiktorowicz, S., and Wolff, S., "Characterizing 51 Eri b from 1 to $5 \mu \mathrm{m}$ : A Partly Cloudy Exoplanet," Astronomical Journal 154, 10 (July 2017).

[11] Konopacky, Q. M., Rameau, J., Duchêne, G., Filippazzo, J. C., Giorla Godfrey, P. A., Marois, C., Nielsen, E. L., Pueyo, L., Rafikov, R. R., Rice, E. L., Wang, J. J., Ammons, S. M., Bailey, V. P., Barman, T. S., Bulger, J., Bruzzone, S., Chilcote, J. K., Cotten, T., Dawson, R. I., De Rosa, R. J., Doyon, R., Esposito, T. M., Fitzgerald, M. P., Follette, K. B., Goodsell, S., Graham, J. R., Greenbaum, A. Z., Hibon, P., Hung, L.-W., Ingraham, P., Kalas, P., Lafrenière, D., Larkin, J. E., Macintosh, B. A., Maire, J., Marchis, F., Marley, M. S., Matthews, B. C., Metchev, S., Millar-Blanchaer, M. A., Oppenheimer, R., Palmer, D. W., Patience, J., Perrin, M. D., Poyneer, L. A., Rajan, A., Rantakyrö, F. T., Savransky, D., Schneider, A. C., Sivaramakrishnan, A., Song, I., Soummer, R., Thomas, S., Wallace, J. K., Ward-Duong, K., Wiktorowicz, S. J., and Wolff, S. G., "Discovery of a Substellar Companion to the Nearby Debris Disk Host HR 2562," Astrophysical Journal Letters 829, L4 (Sept. 2016).

[12] Ingraham, P., Marley, M. S., Saumon, D., Marois, C., Macintosh, B., Barman, T., Bauman, B., Burrows, A., Chilcote, J. K., De Rosa, R. J., Dillon, D., Doyon, R., Dunn, J., Erikson, D., Fitzgerald, M. P., Gavel, D., Goodsell, S. J., Graham, J. R., Hartung, M., Hibon, P., Kalas, P. G., Konopacky, Q., Larkin, J. A., Maire, J., Marchis, F., McBride, J., Millar-Blanchaer, M., Morzinski, K. M., Norton, A., Oppenheimer, R., Palmer, D. W., Patience, J., Perrin, M. D., Poyneer, L. A., Pueyo, L., Rantakyrö, F., Sadakuni, N., Saddlemyer, L., Savransky, D., Soummer, R., Sivaramakrishnan, A., Song, I., Thomas, S., Wallace, J. K., Wiktorowicz, S. J., and Wolff, S. G., "Gemini Planet Imager Spectroscopy of the HR 8799 Planets c and d," Astrophysical Journal Letters 794, L15 (Oct. 2014).

[13] Galicher, R., Rameau, J., Bonnefoy, M., Baudino, J.-L., Currie, T., Boccaletti, A., Chauvin, G., Lagrange, A.-M., and Marois, C., "Near-infrared detection and characterization of the exoplanet HD 95086 b with the Gemini Planet Imager," Astronomy \& Astrophysics 565, L4 (May 2014).

[14] De Rosa, R. J., Rameau, J., Patience, J., Graham, J. R., Doyon, R., Lafrenière, D., Macintosh, B., Pueyo, L., Rajan, A., Wang, J. J., Ward-Duong, K., Hung, L.-W., Maire, J., Nielsen, E. L., Ammons, S. M., Bulger, J., Cardwell, A., Chilcote, J. K., Galvez, R. L., Gerard, B. L., Goodsell, S., Hartung, M., Hibon, 
P., Ingraham, P., Johnson-Groh, M., Kalas, P., Konopacky, Q. M., Marchis, F., Marois, C., Metchev, S., Morzinski, K. M., Oppenheimer, R., Perrin, M. D., Rantakyrö, F. T., Savransky, D., and Thomas, S., "Spectroscopic Characterization of HD 95086 b with the Gemini Planet Imager," Astrophysical Journal 824, 121 (June 2016).

[15] Chilcote, J., Pueyo, L., De Rosa, R. J., Vargas, J., Macintosh, B., Bailey, V. P., Barman, T., Bauman, B., Bruzzone, S., Bulger, J., Burrows, A. S., Cardwell, A., Chen, C. H., Cotten, T., Dillon, D., Doyon, R., Draper, Z. H., Duchêne, G., Dunn, J., Erikson, D., Fitzgerald, M. P., Follette, K. B., Gavel, D., Goodsell, S. J., Graham, J. R., Greenbaum, A. Z., Hartung, M., Hibon, P., Hung, L.-W., Ingraham, P., Kalas, P., Konopacky, Q., Larkin, J. E., Maire, J., Marchis, F., Marley, M. S., Marois, C., Metchev, S., MillarBlanchaer, M. A., Morzinski, K. M., Nielsen, E. L., Norton, A., Oppenheimer, R., Palmer, D., Patience, J., Perrin, M., Poyneer, L., Rajan, A., Rameau, J., Rantakyrö, F. T., Sadakuni, N., Saddlemyer, L., Savransky, D., Schneider, A. C., Serio, A., Sivaramakrishnan, A., Song, I., Soummer, R., Thomas, S., Wallace, J. K., Wang, J. J., Ward-Duong, K., Wiktorowicz, S., and Wolff, S., "1-2.4 $\mu \mathrm{m}$ Near-IR Spectrum of the Giant Planet $\beta$ Pictoris b Obtained with the Gemini Planet Imager," Astronomical Journal 153, 182 (Apr. 2017).

[16] Johnson-Groh, M., Marois, C., De Rosa, R. J., Nielsen, E. L., Rameau, J., Blunt, S., Vargas, J., Ammons, S. M., Bailey, V. P., Barman, T. S., Bulger, J., Chilcote, J. K., Cotten, T., Doyon, R., Duchêne, G., Fitzgerald, M. P., Follette, K. B., Goodsell, S., Graham, J. R., Greenbaum, A. Z., Hibon, P., Hung, L.-W., Ingraham, P., Kalas, P., Konopacky, Q. M., Larkin, J. E., Macintosh, B., Maire, J., Marchis, F., Marley, M. S., Metchev, S., Millar-Blanchaer, M. A., Oppenheimer, R., Palmer, D. W., Patience, J., Perrin, M., Poyneer, L. A., Pueyo, L., Rajan, A., Rantakyrö, F. T., Savransky, D., Schneider, A. C., Sivaramakrishnan, A., Song, I., Soummer, R., Thomas, S., Vega, D., Wallace, J. K., Wang, J. J., Ward-Duong, K., Wiktorowicz, S. J., and Wolff, S. G., "Integral Field Spectroscopy of the Low-mass Companion HD 984 B with the Gemini Planet Imager," Astronomical Journal 153, 190 (Apr. 2017).

[17] Greenbaum, A. Z., Pueyo, L., Ruffio, J.-B., Wang, J. J., De Rosa, R. J., Aguilar, J., Rameau, J., Barman, T., Marois, C., Marley, M. S., Konopacky, Q., Rajan, A., Macintosh, B., Ansdell, M., Arriaga, P., Bailey, V. P., Bulger, J., Burrows, A. S., Chilcote, J., Cotten, T., Doyon, R., Duchêne, G., Fitzgerald, M. P., Follette, K. B., Gerard, B., Goodsell, S. J., Graham, J. R., Hibon, P., Hung, L.-W., Ingraham, P., Kalas, P., Larkin, J. E., Maire, J., Marchis, F., Metchev, S., Millar-Blanchaer, M. A., Nielsen, E. L., Norton, A., Oppenheimer, R., Palmer, D., Patience, J., Perrin, M. D., Poyneer, L., Rantakyrö, F. T., Savransky, D., Schneider, A. C., Sivaramakrishnan, A., Song, I., Soummer, R., Thomas, S., Wallace, J. K., Ward-Duong, K., Wiktorowicz, S., and Wolff, S., "GPI Spectra of HR 8799 c, d, and e from 1.5 to $2.4 \mu \mathrm{m}$ with KLIP Forward Modeling," Astronomical Journal 155, 226 (June 2018).

[18] Crepp, J. R., Principe, D. A., Wolff, S., Giorla Godfrey, P. A., Rice, E. L., Cieza, L., Pueyo, L., Bechter, E. B., and Gonzales, E. J., "GPI Spectroscopy of the Mass, Age, and Metallicity Benchmark Brown Dwarf HD 4747 B," Astrophysical Journal 853, 192 (Feb. 2018).

[19] Rameau, J., Nielsen, E. L., De Rosa, R. J., Blunt, S. C., Patience, J., Doyon, R., Graham, J. R., Lafrenière, D., Macintosh, B., Marchis, F., Bailey, V., Chilcote, J. K., Duchene, G., Esposito, T. M., Hung, L.-W., Konopacky, Q. M., Maire, J., Marois, C., Metchev, S., Perrin, M. D., Pueyo, L., Rajan, A., Savransky, D., Wang, J. J., Ward-Duong, K., Wolff, S. G., Ammons, S. M., Hibon, P., Ingraham, P., Kalas, P., Morzinski, K. M., Oppenheimer, R., Rantakyearö, F. T., and Thomas, S., "Constraints on the Architecture of the HD 95086 Planetary System with the Gemini Planet Imager," Astrophysical Journal Letters 822, L29 (May 2016).

[20] Nielsen, E. L., De Rosa, R. J., Macintosh, B., Wang, J. J., Ruffio, J.-B., Chiang, E., Marley, M. S., Saumon, D., Savransky, D., Ammons, S. M., Bailey, V. P., Barman, T., Blain, C., Bulger, J., Burrows, A., Chilcote, J., Cotten, T., Czekala, I., Doyon, R., Duchêne, G., Esposito, T. M., Fabrycky, D., Fitzgerald, M. P., Follette, K. B., Fortney, J. J., Gerard, B. L., Goodsell, S. J., Graham, J. R., Greenbaum, A. Z., Hibon, P., Hinkley, S., Hirsch, L. A., Hom, J., Hung, L.-W., Dawson, R. I., Ingraham, P., Kalas, P., Konopacky, Q., Larkin, J. E., Lee, E. J., Lin, J. W., Maire, J., Marchis, F., Marois, C., Metchev, S., Millar-Blanchaer, M. A., Morzinski, K. M., Oppenheimer, R., Palmer, D., Patience, J., Perrin, M., Poyneer, L., Pueyo, L., Rafikov, R. R., Rajan, A., Rameau, J., Rantakyrö, F. T., Ren, B., Schneider, A. C., Sivaramakrishnan, A., Song, I., Soummer, R., Tallis, M., Thomas, S., Ward-Duong, K., and Wolff, S., "The Gemini Planet 
Imager Exoplanet Survey: Giant Planet and Brown Dwarf Demographics from 10 to 100 au," Astronomical Journal 158, 13 (July 2019).

[21] Reggiani, M., Quanz, S. P., Meyer, M. R., Pueyo, L., Absil, O., Amara, A., Anglada, G., Avenhaus, H., Girard, J. H., Carrasco Gonzalez, C., Graham, J., Mawet, D., Meru, F., Milli, J., Osorio, M., Wolff, S., and Torrelles, J.-M., "Discovery of a Companion Candidate in the HD 169142 Transition Disk and the Possibility of Multiple Planet Formation," Astrophysical Journal Letters 792, L23 (Sept. 2014).

[22] Currie, T., Cloutier, R., Brittain, S., Grady, C., Burrows, A., Muto, T., Kenyon, S. J., and Kuchner, M. J., "Resolving the HD 100546 Protoplanetary System with the Gemini Planet Imager: Evidence for Multiple Forming, Accreting Planets," Astrophysical Journal Letters 814, L27 (Dec. 2015).

[23] Rapson, V. A., Kastner, J. H., Millar-Blanchaer, M. A., and Dong, R., "Peering into the Giant-planetforming Region of the TW Hydrae Disk with the Gemini Planet Imager," Astrophysical Journal Letters 815, L26 (Dec. 2015).

[24] Long, Z. C., Fernandes, R. B., Sitko, M., Wagner, K., Muto, T., Hashimoto, J., Follette, K., Grady, C. A., Fukagawa, M., Hasegawa, Y., Kluska, J., Kraus, S., Mayama, S., McElwain, M. W., Oh, D., Tamura, M., Uyama, T., Wisniewski, J. P., and Yang, Y., "The Shadow Knows: Using Shadows to Investigate the Structure of the Pretransitional Disk of HD 100453," Astrophysical Journal 838, 62 (Mar. 2017).

[25] Follette, K. B., Rameau, J., Dong, R., Pueyo, L., Close, L. M., Duchêne, G., Fung, J., Leonard, C., Macintosh, B., Males, J. R., Marois, C., Millar-Blanchaer, M. A., Morzinski, K. M., Mullen, W., Perrin, M., Spiro, E., Wang, J., Ammons, S. M., Bailey, V. P., Barman, T., Bulger, J., Chilcote, J., Cotten, T., De Rosa, R. J., Doyon, R., Fitzgerald, M. P., Goodsell, S. J., Graham, J. R., Greenbaum, A. Z., Hibon, P., Hung, L.-W., Ingraham, P., Kalas, P., Konopacky, Q., Larkin, J. E., Maire, J., Marchis, F., Metchev, S., Nielsen, E. L., Oppenheimer, R., Palmer, D., Patience, J., Poyneer, L., Rajan, A., Rantakyrö, F. T., Savransky, D., Schneider, A. C., Sivaramakrishnan, A., Song, I., Soummer, R., Thomas, S., Vega, D., Wallace, J. K., Ward-Duong, K., Wiktorowicz, S., and Wolff, S., "Complex Spiral Structure in the HD 100546 Transitional Disk as Revealed by GPI and MagAO," Astronomical Journal 153, 264 (June 2017).

[26] Kalas, P. G., Rajan, A., Wang, J. J., Millar-Blanchaer, M. A., Duchene, G., Chen, C., Fitzgerald, M. P., Dong, R., Graham, J. R., Patience, J., Macintosh, B., Murray-Clay, R., Matthews, B., Rameau, J., Marois, C., Chilcote, J., De Rosa, R. J., Doyon, R., Draper, Z. H., Lawler, S., Ammons, S. M., Arriaga, P., Bulger, J., Cotten, T., Follette, K. B., Goodsell, S., Greenbaum, A., Hibon, P., Hinkley, S., Hung, L.-W., Ingraham, P., Konapacky, Q., Lafreniere, D., Larkin, J. E., Long, D., Maire, J., Marchis, F., Metchev, S., Morzinski, K. M., Nielsen, E. L., Oppenheimer, R., Perrin, M. D., Pueyo, L., Rantakyrö, F. T., Ruffio, J.-B., Saddlemyer, L., Savransky, D., Schneider, A. C., Sivaramakrishnan, A., Soummer, R., Song, I., Thomas, S., Vasisht, G., Ward-Duong, K., Wiktorowicz, S. J., and Wolff, S. G., "Direct Imaging of an Asymmetric Debris Disk in the HD 106906 Planetary System," Astrophysical Journal 814, 32 (Nov. 2015).

[27] Draper, Z. H., Duchêne, G., Millar-Blanchaer, M. A., Matthews, B. C., Wang, J. J., Kalas, P., Graham, J. R., Padgett, D., Ammons, S. M., Bulger, J., Chen, C., Chilcote, J. K., Doyon, R., Fitzgerald, M. P., Follette, K. B., Gerard, B., Greenbaum, A. Z., Hibon, P., Hinkley, S., Macintosh, B., Ingraham, P., Lafrenière, D., Marchis, F., Marois, C., Nielsen, E. L., Oppenheimer, R., Patel, R., Patience, J., Perrin, M., Pueyo, L., Rajan, A., Rameau, J., Sivaramakrishnan, A., Vega, D., Ward-Duong, K., and Wolff, S. G., "The Peculiar Debris Disk of HD 111520 as Resolved by the Gemini Planet Imager," Astrophysical Journal 826, 147 (Aug. 2016).

[28] Millar-Blanchaer, M. A., Wang, J. J., Kalas, P., Graham, J. R., Duchêne, G., Nielsen, E. L., Perrin, M., Moon, D.-S., Padgett, D., Metchev, S., Ammons, S. M., Bailey, V. P., Barman, T., Bruzzone, S., Bulger, J., Chen, C. H., Chilcote, J., Cotten, T., De Rosa, R. J., Doyon, R., Draper, Z. H., Esposito, T. M., Fitzgerald, M. P., Follette, K. B., Gerard, B. L., Greenbaum, A. Z., Hibon, P., Hinkley, S., Hung, L.-W., Ingraham, P., Johnson-Groh, M., Konopacky, Q., Larkin, J. E., Macintosh, B., Maire, J., Marchis, F., Marley, M. S., Marois, C., Matthews, B. C., Oppenheimer, R., Palmer, D., Patience, J., Poyneer, L., Pueyo, L., Rajan, A., Rameau, J., Rantakyrö, F. T., Savransky, D., Schneider, A. C., Sivaramakrishnan, A., Song, I., Soummer, R., Thomas, S., Vega, D., Wallace, J. K., Ward-Duong, K., Wiktorowicz, S., and Wolff, S., "Imaging an 80 au Radius Dust Ring around the F5V Star HD 157587," Astronomical Journal 152, 128 (Nov. 2016). 
[29] Perrin, M. D., Duchene, G., Millar-Blanchaer, M., Fitzgerald, M. P., Graham, J. R., Wiktorowicz, S. J., Kalas, P. G., Macintosh, B., Bauman, B., Cardwell, A., Chilcote, J., De Rosa, R. J., Dillon, D., Doyon, R., Dunn, J., Erikson, D., Gavel, D., Goodsell, S., Hartung, M., Hibon, P., Ingraham, P., Kerley, D., Konapacky, Q., Larkin, J. E., Maire, J., Marchis, F., Marois, C., Mittal, T., Morzinski, K. M., Oppenheimer, B. R., Palmer, D. W., Patience, J., Poyneer, L., Pueyo, L., Rantakyrö, F. T., Sadakuni, N., Saddlemyer, L., Savransky, D., Soummer, R., Sivaramakrishnan, A., Song, I., Thomas, S., Wallace, J. K., Wang, J. J., and Wolff, S. G., "Polarimetry with the Gemini Planet Imager: Methods, Performance at First Light, and the Circumstellar Ring around HR 4796A," Astrophysical Journal 799, 182 (Feb. 2015).

[30] Esposito, T. M., Fitzgerald, M. P., Graham, J. R., Kalas, P., Lee, E. J., Chiang, E., Duchêne, G., Wang, J., Millar-Blanchaer, M. A., Nielsen, E., Ammons, S. M., Bruzzone, S., De Rosa, R. J., Draper, Z. H., Macintosh, B., Marchis, F., Metchev, S. A., Perrin, M., Pueyo, L., Rajan, A., Rantakyrö, F. T., Vega, D., and Wolff, S., "Bringing "The Moth" to Light: A Planet-sculpting Scenario for the HD 61005 Debris Disk," Astronomical Journal 152, 85 (Oct. 2016).

[31] Dong, R. and Fung, J., "What is the Mass of a Gap-opening Planet?," Astrophysical Journal 835, 146 (Feb. 2017).

[32] Macintosh, B., Chilcote, J. K., Bailey, V. P., de Rosa, R., Nielsen, E., Norton, A., Poyneer, L., Wang, J., Ruffio, J. B., Graham, J. R., Marois, C., Savransky, D., and Veran, J.-P., "The Gemini Planet Imager: looking back over five years and forward to the future," in [Adaptive Optics Systems VI], Close, L. M., Schreiber, L., and Schmidt, D., eds., Society of Photo-Optical Instrumentation Engineers (SPIE) Conference Series 10703, 107030K (July 2018).

[33] Rantakyrö, F. T., Bailey, V. P., Quiroz, C., Chinn, B., Macintosh, B. A., Tallis, M., Millar, B. W., Hayward, T., Poyneer, L., Chilcote, J., Norton, A., and Morrison, C., "Moving the Gemini planet imager to Gemini North: expectations and challenges," in [Ground-based and Airborne Instrumentation for Astronomy VII], Proceedings of the SPIE 10702, in press (2018).

[34] Marley, M. S., Fortney, J. J., Hubickyj, O., Bodenheimer, P., and Lissauer, J. J., "On the Luminosity of Young Jupiters," Astrophysical Journal 655, 541-549 (Jan. 2007).

[35] Burrows, A., Marley, M., Hubbard, W. B., Sudarsky, D., Sharp, C., Lunine, J. I., Guillot, T., Saumon, D., and Freedman, R., "The Spectral Character of Giant Planets and Brown Dwarfs," arXiv.org astro-ph (Sept. 1997).

[36] Fernandes, R. B., Mulders, G. D., Pascucci, I., Mordasini, C., and Emsenhuber, A., "Hints for a Turnover at the Snow Line in the Giant Planet Occurrence Rate," Astrophysical Journal 874, 81 (Mar. 2019).

[37] Skemer, A. J., Marley, M. S., Hinz, P. M., Morzinski, K. M., Skrutskie, M. F., Leisenring, J. M., Close, L. M., Saumon, D., Bailey, V. P., Briguglio, R., Defrere, D., Esposito, S., Follette, K. B., Hill, J. M., Males, J. R., Puglisi, A., Rodigas, T. J., and Xompero, M., "Directly Imaged L-T Transition Exoplanets in the Mid-infrared," Astrophysical Journal 792, 17 (Sept. 2014).

[38] Fitzsimmons, J., Dunn, J., Kerley, D., Lardiere, O., Marois, C., Veran, J.-P., Macintosh, B., Poyneer, L., Madurowicz, A., Chilcote, J., Konopacky, Q., Savransky, D., Maire, J., Esposito, S., Agapito, G., and Bonaglia, M., "GPI 2.0: Design of the pyramid wave front sensor upgrade for GPI," in [Astronomical Telescopes + Instrumentation], Society of Photo-Optical Instrumentation Engineers (SPIE) Conference Series (Dec. 2020).

[39] Agapito, G., Puglisi, A., and Esposito, S., "PASSATA: object oriented numerical simulation software for adaptive optics," in [Adaptive Optics Systems V], Marchetti, E., Close, L. M., and Véran, J.-P., eds., Society of Photo-Optical Instrumentation Engineers (SPIE) Conference Series 9909, 99097E (July 2016).

[40] Madurowicz, A. B., Macintosh, B., Poyneer, L., Veran, J.-P., Ammons, M., Savransky, D., Chilcote, J., Konopacky, Q., De Rosa, R., Marois, C., N'Diaye, M., Perrin, M., Pueyo, L., Soummer, R., LemoineBusserolle, M., and Dupuy, T., "GPI 2.0 : Optimizing reconstructor performance in simulations and preliminary contrast estimates," in [Astronomical Telescopes + Instrumentation], Society of Photo-Optical Instrumentation Engineers (SPIE) Conference Series (Dec. 2020).

[41] Lai, O., Véran, J.-P., Herriot, G., White, J., Ball, J., and Trujillo, C., "Altair performance and upgrades," in [Adaptive Optics Systems IV], Marchetti, E., Close, L. M., and Vran, J.-P., eds., Society of Photo-Optical Instrumentation Engineers (SPIE) Conference Series 9148, 914838 (July 2014). 
[42] Poyneer, L. A., Palmer, D. W., Macintosh, B., Savransky, D., Sadakuni, N., Thomas, S., Véran, J.-P., Follette, K. B., Greenbaum, A. Z., Mark Ammons, S., Bailey, V. P., Bauman, B., Cardwell, A., Dillon, D., Gavel, D., Hartung, M., Hibon, P., Perrin, M. D., Rantakyrö, F. T., Sivaramakrishnan, A., and Wang, J. J., "Performance of the Gemini Planet Imager's adaptive optics system," Applied Optics 55, 323 (Jan. 2016).

[43] Soummer, R., "Apodized Pupil Lyot Coronagraphs for Arbitrary Telescope Apertures," Astrophysical Journal Letters 618, L161-L164 (Jan. 2005).

[44] Soummer, R., Pueyo, L., Ferrari, A., Aime, C., Sivaramakrishnan, A., and Yaitskova, N., "Apodized Pupil Lyot Coronagraphs for Arbitrary Apertures. II. Theoretical Properties and Application to Extremely Large Telescopes," Astrophysical Journal 695, 695-706 (Apr. 2009).

[45] Soummer, R., Sivaramakrishnan, A., Pueyo, L., Macintosh, B., and Oppenheimer, B. R., "Apodized Pupil Lyot Coronagraphs for Arbitrary Apertures. III. Quasi-achromatic Solutions," Astrophysical Journal 729, 144 (Mar. 2011).

[46] N'Diaye, M., Pueyo, L., and Soummer, R., "Apodized Pupil Lyot Coronagraphs for Arbitrary Apertures. IV. Reduced Inner Working Angle and Increased Robustness to Low-order Aberrations," Astrophysical Journal 799, 225 (Feb. 2015).

[47] Zimmerman, N. T., Eldorado Riggs, A. J., Jeremy Kasdin, N., Carlotti, A., and Vanderbei, R. J., "Shaped pupil Lyot coronagraphs: high-contrast solutions for restricted focal planes," Journal of Astronomical Telescopes, Instruments, and Systems 2, 011012 (Jan. 2016).

[48] Fogarty, K., Mazoyer, J., St. Laurent, K., Soummer, R., N’Diaye, M., Stark, C., and Pueyo, L., "Optimal deformable mirror and pupil apodization combinations for apodized pupil Lyot coronagraphs with obstructed pupils," in [Society of Photo-Optical Instrumentation Engineers (SPIE) Conference Series], 10698, 106981J (Aug. 2018).

[49] Marois, C., Gerard, B., Lardière, O., Thompson, W., and Véran, J.-P., "Upgrading the Gemini Planet Imager calibration unit with a photon counting focal plane wavefront sensor," in [Astronomical Telescopes + Instrumentation], Society of Photo-Optical Instrumentation Engineers (SPIE) Conference Series (Dec. 2020).

[50] Limbach, M. A., Chilcote, J., Konopacky, Q., De Rosa, R., Hamper, R., Macintosh, B., Marois, C., Perrin, M., Savransky, D., Veran, J.-P., Wang, J., and Aleman, A., "GPI 2.0: Upgrades to the IFS including new spectral modes," in [Astronomical Telescopes + Instrumentation], Society of Photo-Optical Instrumentation Engineers (SPIE) Conference Series (Dec. 2020).

[51] Groff, T., Chilcote, J., Brandt, T., Kasdin, N. J., Galvin, M., Loomis, C., Rizzo, M., Knapp, G., Guyon, O., Jovanovic, N., Lozi, J., Currie, T., Takato, N., and Hayashi, M., "First light of the CHARIS high-contrast integral-field spectrograph," in [Society of Photo-Optical Instrumentation Engineers (SPIE) Conference Series], Society of Photo-Optical Instrumentation Engineers (SPIE) Conference Series 10400, 1040016 (Sept. 2017). 